Editorial

\title{
Acute undifferentiated fever in children- an overview
}

\author{
Rabindran ${ }^{1}$, Verma $\mathbf{M}^{2}$ \\ ${ }^{1}$ Dr. Rabindran, Consultant, Neonatologist, Billroth Hospital, Chennai, India, ${ }^{2}$ Mrs Mamta Verma, Associate \\ Professor, AIIMS College of Nursing, Bhopal, MP, India.
}

Address for Correspondence: Dr. Rabindran, E- mail: rabindranindia@yahoo.co.in

\begin{abstract}
Fever is one of the common reason for OPD as well as in patient admission department. Due to improvements of laboratory facilities \& imaging the incidence and etiological profile of fever have drastically changed. Proper history taking and systematic examination remains the gold standard for fever evading diagnosis.
\end{abstract}

Keywords: Acute Undifferentiated fever, Pyrexia of unknown origin, Children

Acute Undifferentiated fever (AUF) is defined as measured temperature $\geq 38^{\circ} \mathrm{C}$ and history of febrile illness of 2-14 days duration, with no localized cause as judged by the treating physician [1]. They can be associated with abdominal pain, diarrhoea, haematochezia, nausea or vomiting, rhinorrhoea, breathlessness, ocular pain, altered sensorium, headache, neck stiffness, rash, joint pain, muscle ache, petechiae, ecchymosis, nose or gum bleeding and jaundice. AUF is different from pyrexia of unknown origin- fever of at least 3 weeks with no identified cause even after investigation [2].

Due to improvements of laboratory facilities \& imaging the incidence and etiological profile of fever have drastically changed. Proper history taking and systematic examination remains the gold standard for fever evading diagnosis. Specially for infants under one month of age who are at risk for serious and rapidly progressive bacterial and viral infections judicious protocol based investigation and pre-emptive therapies are mandatory [3]. A syndromic approachto tropical infections can help in arriving at an etiology, plan investigation panel and choose early rational empiric therapy [4].

Tropical fever can be broadly classified as 1) Undifferentiated fever \{Malaria ( $\mathrm{P}$. falciparum), scrub typhus, leptospirosis, typhoid, dengue $\} ; 2$ ) Fever with rash/ thrombo cytopenia \{Dengue, rickettsial infections, meningococcal infection, malaria (falciparum), leptospirosis, measles, rubella\}; 3) Fever with ARDS \{Scrub typhus, falciparum malaria, influenza - H1N1, leptospirosis, hantavirus, meliodosis, Legionella spp., Streptococcus pneumoniae\}; 4) Febrile encephalopathy \{Herpes simplex virus, Japanese B, S. pneumoniae, Neisseria meningitidis, Haemophilus influenzae, enteroviruses, scrub typhus, cerebral malaria and typhoid encephalopathy $\}$; 5) Fever with multiorgan dysfunction \{falciparum malaria, leptospirosis, scrub typhus, dengue, hepatitis A or E, Hanta virus infection $\}[5]$.

Complete investigation panel should include tests for common and rare diseases. Malaria should be ruled out by peripheral blood smear with Giemsa stain, Immuno-chromatographic test to detect lactate dehydrogenase (LDH) for Plasmodium falciparum and Plasmodium vivax and HRP2 to detect Plasmodium falciparum.

For dengue and chikun-gunya virus infections ELISA with specific IgM antibodies needs to be done. Enteric fever should be ruled out by enzyme immunoassay to detectIgM and IgG antibodies. Widal test should include agglutinating antibodies against $\mathrm{O}$ and $\mathrm{H}$ antigens of $\mathrm{S}$. typhi and $\mathrm{H}$ antigens of S. paratyphi A [6]

In developing country like india the diagnostic panel for acute undifferentiated fever includes first line investigations like Malaria microscopy, blood culture, Dengue rapid NS1 antigen and IgM Combo test, Leptospira IgM ELISA, Scrub typhus IgM ELISA and Chikungunya IgM ELISA. Second line 


\section{Editorial}

testing includes Dengue IgM capture ELISA (MACELISA), Scrub typhus immunofluorescence (IFA), Leptospira Microscopic Agglutination Test (MAT), malaria PCR and malaria immunochromatographic rapid diagnostic test (RDT)[1].

Case definitions of common causes of undifferentiated fever are: Leptospirosis- Positive ELISA \& MAT; Scrub typhus- Positive ELISA \& IFA; Dengue - Positive RDT \& /or positive MACELISA;

Chikungunya: Positive ELISA; Bacteraemia: Growth of bacteria other than contaminants in blood culture; Malaria- Positive malaria genus-specific PCR. Coinfections must be differentiated from false positive reports.

Treatment of AUF needs clinical discretion of etiology. Majority are treated with empirical antimicrobials. Chloroquine may be started for suspected malaria, Doxycycline or Azithromycin for scrub typhus, Ceftriaxone for Enteric fever. Dengue needs symptomatic management with fluids. Ceftriaxone or Amikacin may be started for suspected Urinary tract infection \& Ampicillin with Gentamycin or Amoxicillin alone for lower respiratory tract infection.

The etiology of AUFI remains unknown even after strenuous efforts in many cases. Vector control measures, drinking water supply and sanitation should be improved to prevent vector borne and water borne diseases [7].

Region specific epide-miological database of causes of AUFI needs to be created. Rational, standardized protocol based assessment and treatment of children with acute undifferentiated fever can reduce unwanted investigations and antimicrobial use.

Funding: Nil, Conflict of interest: None initiated, Perission from IRB: Yes

\section{References}

1. Kristine Mørch, Anand Manoharan, Sara Chandyet al., Acute undifferentiated fever in India: a multicentre study of aetiology and diagnostic accuracy. BMC Infect Dis. Oct 2017;17:665.doi: 10. 1186/s12879-017-2764-3

2. Nield LS, Kamat D. Fever without focus. Kliegman RM, Stanton BF, Schor NF, St Geme JW, Behrman RE, editors. Nelson textbook of pediatrics. 20th ed. Philadelphia, PA: Elsevier Inc.; 2016. p. 1283.

3. Long SS. Diagnosis and management of undifferentiated fever in children. J Infect. 2016 Jul 5;72 Suppl:S68-76. doi: 10.1016/j.jinf.2016.04.025. Epub 2016 May 18.

4. SunitSinghi, Dhruva Chaudhary, George M. Varghese et al., Tropical fevers: Management guidelines. Indian J Crit Care Med. 2014 Feb; 18(2): 62-69. doi:10.4103/0972-5229.126074.

5. Frean J, Blumberg L. Ch 5A. Brisbane: ACTM Publication; 2005. Tropical fevers part A. Viral, bacterial and fungal infections Primer of Tropical Medicine; pp. 1-18.

6. Balvinder Singh Arora, Monika Matlani, Karnika Saigal, Indu Biswal, Santhosh Rajan, Arun Padmanandan, Saudan Singh. Major aetiologies of acute undifferentiated fever in 2013 and 2014: an experience in retrospect. DOI: http://dx.doi.org/10. 18203/2349-3933.ijam20171062.

7. Gowri Veligandla, Ezhilvanan, E. Padmavathi and Bhaskar, M. 2017. Etiological Spectrum and Prevalence of Acute Undifferentiated Febrile Illness (AUFI) in Fever Cases Attending our Tertiary Care Centre. Int.J.Curr.Microbiol.App.Sci. 6(5): 954-962. doi: https://doi.org/10.20546/ijcmas.2017.605.105.

\section{How to cite this article?}

Rabindran, Verma M. Acute undifferentiated fever in children- an overview. Int J Pediatr Res. 2017;4(11):634635.doi:10. 17511/ijpr.2017.i11.01. 